\title{
Efecto del extracto hidroalcohólico del fruto de granada (Punica granatum L.) presentada en forma de gel farmacéutico en el tratamiento de las hemorroides
}

\section{Effect of Hydro-Alcoholic Extract of the Fruit of Pomegranate (Punica granatum L.) in Pharmaceutical Gel for the Treatment of Hemorrhoids}

\author{
Carlos Alejandro Bell Cortez ${ }^{1}$
}

http://dx.doi.org/10.21503/CienciayDesarrollo.2015.v18i1.02

\section{RESUMEN}

Se evaluó el efecto de gel formulado a base de extracto hidroalcohólico del fruto de la granada (Punica granatum L.), sobre las hemorroides, en 50 voluntarios de ambos sexos con edades comprendidas entre 20 y 50 años, todos residentes en la ciudad de Lima (Perú). La aplicación y seguimiento farmacológico se realizó dos veces al día durante un mes; la dosis varió según el tamaño de la hemorroides; se excluyó la utilización de cualquier otro preparado convencional. El ensayo, previo consentimiento informado, mostró tener efecto en el proceso de desinflamación del tejido comprometido por las hemorroides. El 46,67 $\%$ fue evaluado como excelente, el 31,11\% como bueno, el 20,00 \% como regular y el 2,22 \% como nulo. Se concluye que el fruto de la granada es una fuente de materia prima natural, de bajo costo, que eleva la calidad de atención en enfermería en el tratamiento de las hemorroides

Palabras clave: gel de granada, hemorroides, desinflamación.

\section{ABSTRACT}

The effect of gel formulated with hydroalcoholic extract of the fruit of the pomegranate (Punica granatum L.) on hemorrhoids in 50 male and female volunteers aged between 20 and 50 years, all whom resident in Lima (Peru). The application and monitoring of the pharmaceutical was carry out twice a day for a month, the dose varied according to the size of the hemorrhoids, the use all any conventional preparation was excluded. The trial, previously consent, was shown to have an effect on reducing inflammation in tissue compromised by the hemorrhoids. The $46,67 \%$ the cases was rated excellent, $31,11 \%$ was rated good and 20,00 \% was rated fair and 2,22\% shown no effect. We conclude that the fruit of the pomegranate is a source of natural raw material of low cost, which raises the quality of nursing care in the treatment of hemorrhoids.

Key words: pomegranate, hemorrhoids, reducing inflammation.

1 Doctor en Farmacia y Bioquímica, docente principal de la UNMSM, investigador del Instituto de Ciencias Farmacéuticas y Recursos Naturales “Juan de Dios Guevara”, UNMSM. Docente de la asignatura de Farmacología de la Escuela Profesional de Enfermería de la Facultad de Medicina Humana y Ciencias de la Salud de la UAP. E-mail: carlosbellcortez@yahoo.es 


\section{INTRODUCCIÓN}

Las hemorroides, llamadas también almorranas, son dilataciones e inflamaciones de las venas del recto y del ano. La principal causa es el estreñimiento, que generalmente se origina por una dieta deficiente, falta de ejercicio físico, estrés y escasa ingesta de líquidos

El fruto que se emplea en el presente estudio: granada (Punica granatum L.), proviene de un árbol pequeño, cuyo pericarpio es de color amarillo a rojizo, de pulpa ácida y con una gran cantidad de semillas; pertenece a la familia Punicaceae [1].

El conocimiento popular le atribuye a este fruto, propiedades antiinflamatorias, antibióticas y antivirales [2-4]. En la presente investigación, se valida el conocimiento del empleo del zumo del fruto de granada (Punica granatum $L$.$) en el tratamiento de las hemorroides; el$ estudio se realizó empleando el extracto hidroalcohólico del fruto, presentado en forma de gel farmacéutico

\section{HIPÓTESIS}

El extracto hidroalcohólico del fruto de la granada (Punica granatum L.), aplicado en forma de gel farmacéutico, tiene efecto antiinflamatorio sobre las hemorroides

\section{MÉTODOS Y RESULTADOS}

\section{Material:}

A. Reactivos: Etanol absoluto, ácido poliacrílico, alcohol isopropílico, propilenglicol, Edta, preservantes, agua destilada estéril.

B. Equipos: Espectrofotómetro UV-Visible, Marca Thermo Scientific, Modelo HeliosZeta; Cromatógrafo de gases Perkin Elmer modelo Clarus 600; Espectrómetro Perkin
Elmer modelo Spectrum 100N; Balanza Analítica Marca Ohaus Modelo Adventurer; Equipo emulsificador de laboratorio; Potenciómetro; Termómetro digital. Estufa Marca Labor Musze Ripari.

\section{Metodología:}

A. Clasificación taxonómica, recolección y preparación del extracto

REINO: Plantae

DIVISIÓN: Magnoliophyta

CLASE: Magnoliopsida

ORDEN: Myrtales

FAMILIA: Punicaceae

GÉNERO: Punica

ESPECIE: Punica granatum $L$.

Se seleccionaron frutos de granada (Punica granatum $L$.) de un huerto ubicado en el distrito de Lurín (Lima-Perú).

Se procedió a lavarlas, secarlas y retirarle la cáscara; luego la parte comestible se licuó con alcohol etílico de $70^{\circ}$ en la proporción 1x3 (P/P). Se dejó macerar por quince días en oscuridad para la extracción de los metabolitos. Luego, se filtró y se obtuvo una solución rojiza que se evaporó en estufa de aire circulante a temperatura no superior a $40{ }^{\circ} \mathrm{C}$, obteniéndose un extracto blando de color marrón.

B. Análisis físico químico del extracto de granada (Punica granatum L.)

Se efectuaron los siguientes análisis físicos: aspecto, color olor, sabor, $\mathrm{pH}$, humedad [5].

Análisis químico: cenizas. 


\section{Formulación y estabilidad del gel}

Se ensayó la formulación que se describe, para lo cual se tuvo en cuenta que los ingredientes seleccionados sean compatibles con el extracto obtenido y que, sobre todo, sean estables en el tiempo así como de bajo costo:

Extracto de granada $5,00 \mathrm{~g}$

Agentes gelificantes, diluyentes y preservantes $7,70 \mathrm{~g}$

Agua destilada estéril c.s.p $100,00 \mathrm{~g}$

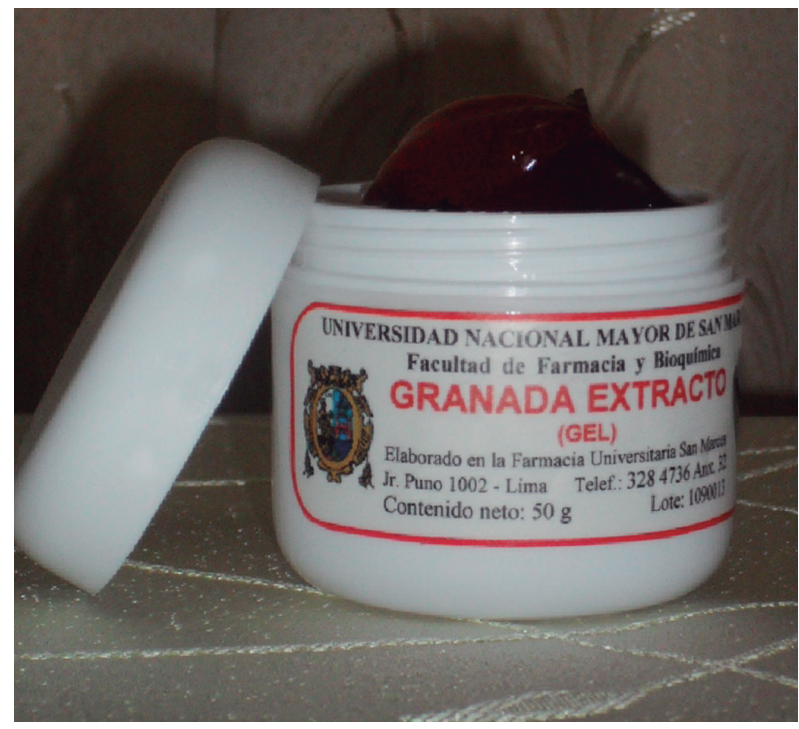

Figura 1. Gel formulado con granada.

\section{Ensayo de estabilidad acelerada de la formulación.}

Con el fin de determinar la estabilidad del preparado y controlar sus posibles alteraciones, se efectuaron ensayos de estabilidad acelerada, sometiendo la formulación a diferentes temperaturas. Para realizar esta prueba, se procedió a evaluar cada quince días, hasta completar tres meses, los siguientes parámetros: aspecto, consistencia, color, olor y $\mathrm{pH}$.

\section{Ensayo efectuado en voluntarios}

Participaron en el estudio, 50 voluntarios de ambos sexos, con edades comprendidas entre 50 y 70 años, que presentaban hemorroides visibles; todos residentes de diversos distritos de Lima Metropolitana.

Se dividió a los participantes en dos grupos:

Grupo I (grupo problema - 45 pacientes): Se administró gel con extracto de granada.

Grupo II (grupo control - 5 pacientes): Se administró gel sin extracto de granada.

Previo consentimiento escrito, se procedió a la aplicación del gel a base de extracto de granada a los pacientes voluntarios, con hemorroides dos veces al día, practicándose previamente un lavado con agua tibia.

El estudio se extendió durante 30 días y se administró una dosis de preparado que dependió del tamaño de la hemorroide, excluyendo la utilización de cualquier otro preparado convencional. El seguimiento farmacológico se efectúo todos los días, lo que se documentó con evidencia fotográfica.

\section{RESULTADOS}

\section{MARCHA FITOQUÍMICA DEL EXTRACTO HIDROALCOHÓLICO DE GRANADA (Punica granatum L.)}

Se determinó la presencia de:

Taninos

Triterpenoides

Flavonoides 


\section{A. ANÁlisis FISICOQUÍMICO DEL EXTRACTO HIDROALCOHÓLICO DE GRANADA (Punicagranatum L.)}

Tabla 1. Protocolo de análisis del extracto de granada

\begin{tabular}{|c|c|c|}
\hline PRODUCTO & : & Extracto de granada (Punica granatum L.) \\
\hline CANTIDAD & : & $50 \mathrm{~g}$ \\
\hline FECHA de preparación de extracto & : & Mayo de 2013 \\
\hline FECHA DE ANÁLISIS & : & Mayo de 2013 \\
\hline DETERMINACIONES & & RESULTADOS \\
\hline \multicolumn{3}{|l|}{ A) FÍSICAS: } \\
\hline ASPECTO & & Masa blanda \\
\hline COLOR & & Marrón claro \\
\hline SABOR & & Ligeramente dulce \\
\hline OLOR & & Sui géneris. \\
\hline $\mathrm{pH}$ (solución acuosa $10 \%-25^{\circ} \mathrm{C}$ ) & & 2,10 \\
\hline HUMEDAD & & $2,81 \%$ \\
\hline \multicolumn{3}{|l|}{ B) QUÍMICAS: } \\
\hline CENIZAS TOTALES & & $2,53 \%$ \\
\hline
\end{tabular}

\section{B. EVALUACIÓN FARMACOTÉCNICA DEL GEL}

\begin{tabular}{ll}
\multicolumn{1}{c}{ FICHA TÉCNICA DE ESTABILIDAD DEL PRODUCTO TERMINADO } \\
\hline PRODUCTO & Extracto de granada (Punica granatum L.) \\
\hline FORMA FARMAC. & Gel \\
\hline PRESENTACIÓN & Frasco $x 0 \mathrm{~g}$. \\
\hline No DE LOTE & Piloto No 001 \\
\hline FECHA FAB. & Mayo 2013 \\
\hline FECHA VENC. & Mayo 2013 \\
\hline
\end{tabular}

Tabla 2. Resultados

\begin{tabular}{|c|c|c|c|c|c|}
\hline \multirow{2}{*}{ DETERMINACIONES } & \multirow{2}{*}{ ESPECIFICACIONES } & \multicolumn{4}{|c|}{ RESULTADOS } \\
\hline & & Inicio & 1 mes & 2 meses & 3 meses \\
\hline ASPECTO & Gel translúcido & Cumple & Cumple & Cumple & Cumple \\
\hline COLOR & Marrón anaranjado & Cumple & Cumple & Cumple & Cumple \\
\hline OLOR & Sui géneris & Cumple & Cumple & Cumple & Cumple \\
\hline VISCOSIDAD & $100000-150000 \mathrm{cps}$ & 130000 & 120000 & 120000 & 120000 \\
\hline $\mathrm{pH}$ & $5,5-7,0$ & 6,0 & 5,9 & 5,9 & 5,9 \\
\hline \multicolumn{6}{|l|}{ RECUENTO MICROBIANO } \\
\hline AEROBIOS MESOFILOS & Menos de $100 \mathrm{ufc} / \mathrm{g}$ & Conforme & Conforme & Conforme & Conforme \\
\hline RECUENTO DE HONGOS & Menos de $10 \mathrm{ufc} / \mathrm{g}$ & Conforme & Conforme & Conforme & Conforme \\
\hline RECUENTO DE LEVADURAS & Menos de $10 \mathrm{ufc} / \mathrm{g}$ & Conforme & Conforme & Conforme & Conforme \\
\hline ESCHERICHIA COLI & Ausente en $1 \mathrm{~g}$ & Conforme & Conforme & Conforme & Conforme \\
\hline SALMONELLA spp & Ausente en $1 \mathrm{~g}$ & Conforme & Conforme & Conforme & Conforme \\
\hline STAPHYLOCOCCUS AUREUS & Ausente en $1 \mathrm{~g}$ & Conforme & Conforme & Conforme & Conforme \\
\hline PSEUDOMONAS AERUGINOSA & Ausente en $1 \mathrm{~g}$ & Conforme & Conforme & Conforme & Conforme \\
\hline
\end{tabular}




\section{EVALUACIÓN FARMACOLÓGICA}

Tabla 3. Valoración farmacológica por semanas

\begin{tabular}{|c|c|c|c|c|c|c|c|c|c|c|c|c|c|c|c|c|}
\hline & \multicolumn{4}{|c|}{$1^{\circ}$ Semana } & \multicolumn{4}{|c|}{$2^{\circ}$ Semana } & \multicolumn{4}{|c|}{$3^{\circ}$ Semana } & \multicolumn{4}{|c|}{$4^{\circ}$ Semana } \\
\hline & \multicolumn{2}{|c|}{ GRUPO I } & \multicolumn{2}{|c|}{ GRUPO II } & \multicolumn{2}{|c|}{ GRUPO I } & \multicolumn{2}{|c|}{ GRUPO II } & \multicolumn{2}{|c|}{ GRUPO I } & \multicolumn{2}{|c|}{ GRUPO II } & \multicolumn{2}{|c|}{ GRUPO I } & \multicolumn{2}{|c|}{ GRUPO II } \\
\hline & $\mathbf{N}^{\circ}$ & $\%$ & $\mathbf{N}^{\circ}$ & $\%$ & $\mathbf{N}^{\circ}$ & $\%$ & $\mathbf{N}^{o}$ & $\%$ & $\mathbf{N}^{o}$ & $\%$ & $\mathbf{N}^{o}$ & $\%$ & $\mathbf{N}^{o}$ & $\%$ & $\mathbf{N}^{\circ}$ & $\%$ \\
\hline Excelentes & - & - & - & - & - & - & - & - & 14 & 31,10 & - & - & 21 & 46,67 & - & - \\
\hline Buenos & - & - & - & - & 18 & 40,00 & - & - & 12 & 26,70 & - & - & 14 & 31,11 & - & - \\
\hline Regulares & 2 & 4,44 & - & - & 16 & 35,55 & - & - & 13 & 28,90 & - & - & 9 & 20,00 & - & - \\
\hline Nulo & 43 & 95,56 & 5 & 100,0 & 11 & 24,45 & 5 & 100,0 & 6 & 13,30 & 5 & 100,0 & 1 & 2,22 & 5 & 100,0 \\
\hline Total & 45 & 100,0 & 5 & 100,0 & 45 & 100,0 & 5 & 100,0 & 45 & 100,0 & 5 & 100,0 & 45 & 100,0 & 5 & 100,0 \\
\hline
\end{tabular}

De acuerdo con la tabla calificadora de resultados de Balbuena Gonzales y Chapano Jairo (1987), la evaluación clínica corresponde a la clasificación que se señala en la tabla 4

Tabla 4

\begin{tabular}{lcc}
\multicolumn{1}{c}{ CALIFICACIÓN } & $\mathbf{N}^{\circ}$ casos & $\%$ \\
\hline $\begin{array}{l}\text { EXCELENTES } \\
\text { (reducción entre 80-100\%) }\end{array}$ & 21 & 46,67 \\
$\begin{array}{l}\text { BUENOS } \\
\text { (reducción entre el 60-80\%) }\end{array}$ & 14 & 31,11 \\
\hline $\begin{array}{l}\text { REGULARES } \\
\text { (reducción entre 40-60\%) }\end{array}$ & 9 & 20,00 \\
$\begin{array}{l}\text { NULO } \\
\text { (reducción menor de un 40\%) }\end{array}$ & 1 & 2,22 \\
\hline $\begin{array}{l}\text { SUBTOTAL } \\
\text { “GRUPO CONTROL.” }\end{array}$ & 20 & 100 \\
\hline
\end{tabular}

\section{EVOLUCIÓN CLÍNICA}

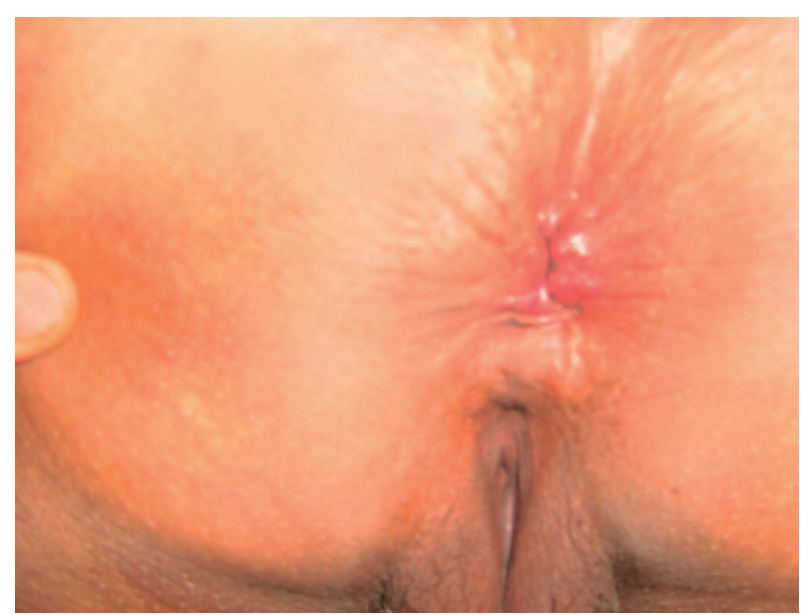

Antes

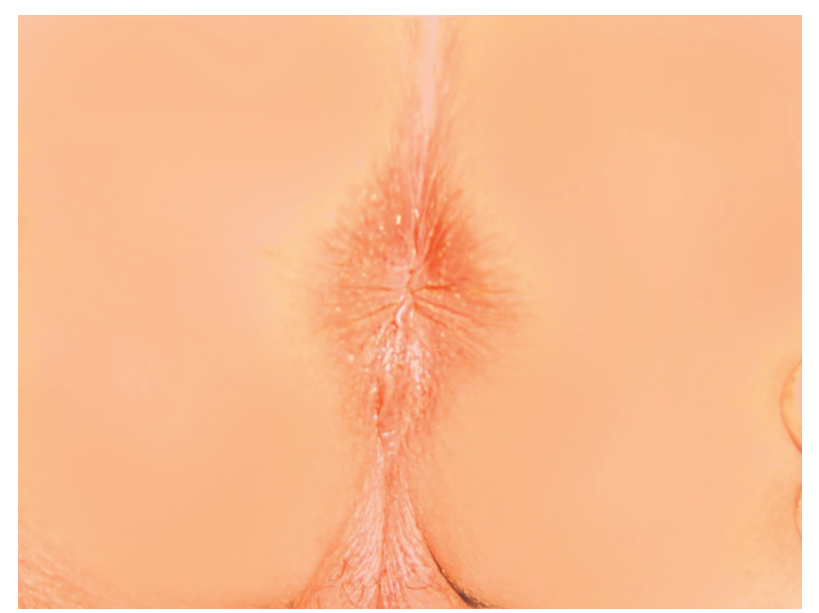

Después 


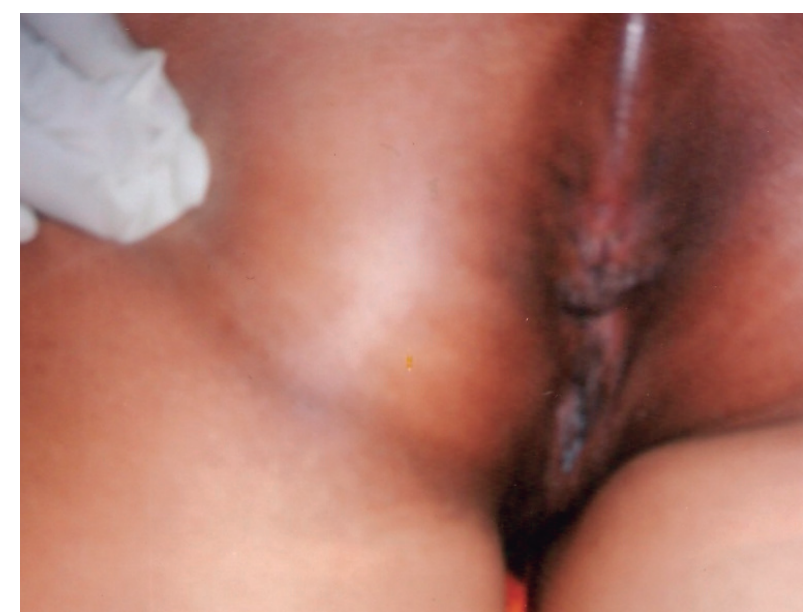

Antes

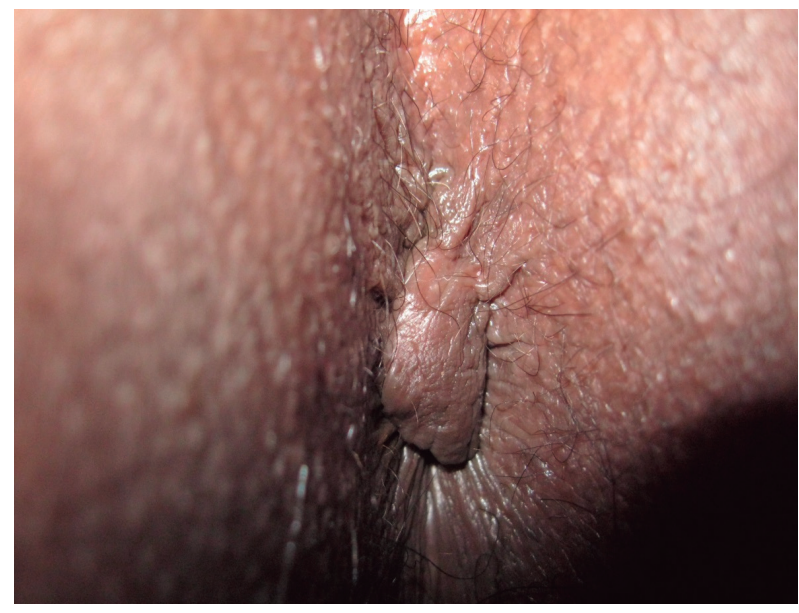

Antes

\section{ANÁLISIS Y DISCUSIÓN}

Los resultados obtenidos, muestran una marcada acción favorable de parte del gel (figura 1) formulado a base del extracto hidroalcohólico del fruto de la granada (Punica granatum L.), en el proceso de desinflamación del tejido comprometido por la úlcera por la hemorroide (tablas 3 y 4 ).

Durante la evaluación diaria, se observó que no hubo alergias en el sitio de aplicación ni complicaciones visibles [6] por lo que se puede decir, en términos generales, que el ensayo tuvo una evolución satisfactoria. La marcha fitoquímica mostró que el extracto contiene una cantidad importante de Flavonoides [7] cuyo efecto antiinflamatorio es muy bien conocido; así mismo,

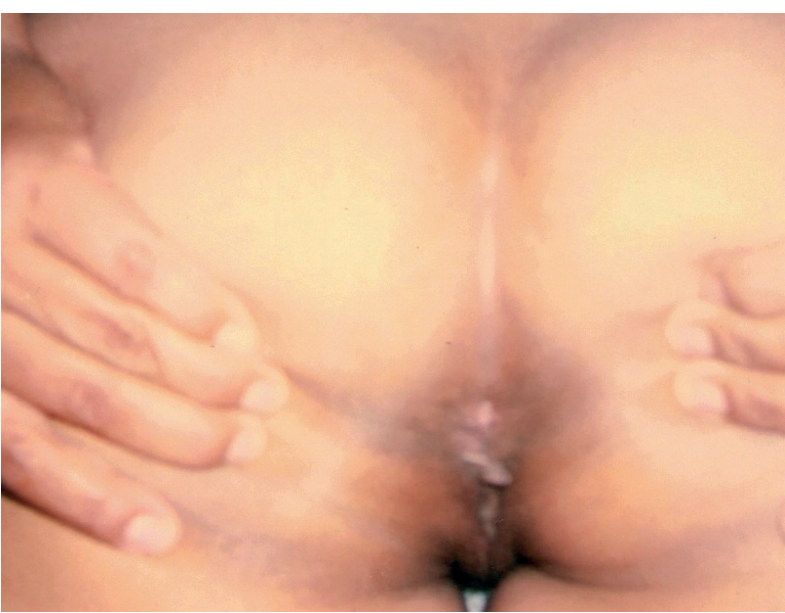

Después

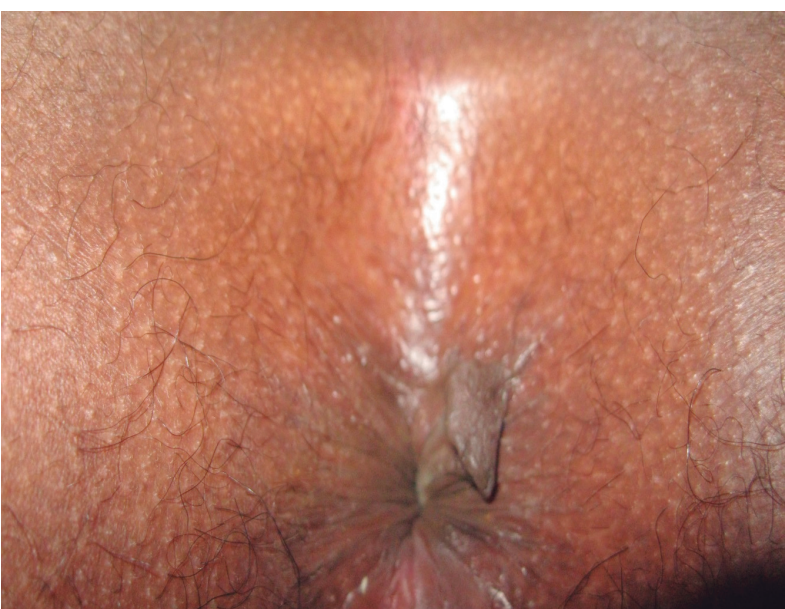

Después

los taninos que contiene le confiere al extracto propiedades astringentes y antiinflamatorias. El estudio de estabilidad acelerado practicado al gel farmacéutico, demostró que este es estable y perfectamente compatible con el extracto hidroalcohólico, no evidenciándose cambios organolépticos ni fisicoquímicos, por lo que se puede afirmar que tendría un tiempo de vida media de tres años.

El estudio farmacológico, llevado a cabo con 50 voluntarios, dejó la evidencia de que el extracto hidroalcohólico del fruto de la granada administrado en forma de gel (Fig. 1), tiene efecto en el proceso de desinflamación del tejido afectado, debido posiblemente al efecto sinérgico de los metabolitos flavonoides + taninos. 


\section{CONCLUSIONES}

1. El extracto hidroalcohólico del fruto de granada (Punica granatum L.), administrado en forma de gel, mostró tener efecto antiinflamatorio sobre las hemorroides

2. De acuerdo con la escala calificadora de Balbuena Gonzales y Chapano Jairo, el 46,67 $\%$ fue evaluado como excelente, el 31,11\% como bueno y el $20,00 \%$ como regular y el $2,22 \%$ como nulo.

3. Por su fácil adquisición, bajo costo y ser una sustancia de origen natural, el extracto hidroalcohólico del fruto de la granada (Punica granatum L.) puede constituir un importante activo para su aplicación en el tratamiento de las hemorroides

\section{REFERENCIAS}

1. Bonsaimania. 2009. Granado (Punica granatum). 06 de julio 2009. http://bonsaimania.com/Bonsái_ chas/punica_granatum.htm.

2. Naz S, Siddigi R, Ahmad S, Rasool SA, Sayeed SA. Antibacterial activity directed isolation of com- pounds from Punica granatum.J Food Sci. 2007; 9: 341-345.

3. Schubert SY, Lansky EP, Neeman I. Antioxidant and eicosanoid enzyme inhibition properties of pomegranate seed oil and fermented juice _ avonoids. J Ethnopharmacol. 1999; 66: 11-17. CoraoMéndez y col./Rev Fac Farm. 2009; 51 (1): 15-19

4. Nuerath AR, Strick N, Li YY, Debnath AK. Punica granatum (pomegranate) juice provides and HIV1 entry inhibitor and candidate topical microbicide. Ann N Y Acad Sci. 2005; 1056: 311-327.

5. British Herbal Pharmacopoeia. (1990) Vol. I. Bournemouth, Dorset: British Herbal Medical Association, pp. 69-70.

6. Vidal A, Fallarero A, Pena BR, Medina ME, Gra B, Rivera F, Gutierrez Y, Vuorela PM. Studies on the toxicity of Punica granatum L. (Punicaceae) whole fruit extracts. J Ethnopharmacol. 2003; 89: 295-300.

7. Méndez-Corao G, Cova JA, Pérez J. Efecto antiestrés del extracto del pericarpio de la Punica granatum $\mathrm{L}$ en células mononucleares humanas de sangre periférica. Ciencia. 2004; 12: 276-282. 